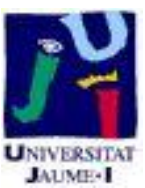

Título artículo / Títol article: On the solvability of groups with four class sizes

Autores / Autors Antonio Beltrán, María José Felipe

Revista:

Journal of Algebra and Its Applications (2012), 11:06

Versión / Versió:

Postprint del autor

Cita bibliográfica / Cita

BELTRÁN, Antonio; FELIPE, María José. On the bibliogràfica (ISO 690): solvability of groups with four class sizes. Journal of Algebra and Its Applications, 2012, vol. 11, no.06, p. $1250036-43$

url Repositori UJI:

http://hdl.handle.net/10234/69200 


\title{
ON THE SOLVABILITY OF GROUPS WITH FOUR CLASS SIZES.
}

\author{
Antonio Beltrán \\ Departamento de Matemáticas, \\ Universidad Jaume I, 12071 Castellón, Spain \\ e-mail: abeltran@mat.uji.es \\ and \\ María José Felipe \\ Instituto Universitario de Matemática Pura y Aplicada, \\ Universidad Politécnica de Valencia, \\ 46022 Valencia, Spain \\ e-mail: mfelipe@mat.upv.es
}

\begin{abstract}
It is shown that if the set of conjugacy class sizes of a finite group $G$ is $\{1, m, n, m n\}$, where $m, n$ are positive integers which do not divide each other, then $G$ is up to central factors a $\{p, q\}$-group. In particular, $G$ is solvable.
\end{abstract}

\section{Introduction}

It is known that the structure of a finite group is strongly controlled by the set of its conjugacy class sizes. For example, groups with two class sizes are nilpotent and groups with three class sizes are solvable. However, in general groups with four class sizes are not solvable, and even may be simple, as $\operatorname{PSL}(2,5)$. The authors showed in [2] and [3] that if the set of conjugacy class sizes of a group $G$ is exactly $\{1, m, n, m n\}$ with $m$ and $n$ realtively prime integers, then $G$ is solvable and in fact, it is nilpotent and $m$ and $n$ are prime powers. A. Camina and R. Camina have recently proved in [5] that groups having four class sizes such that two of them are coprime numbers are also solvable. Furthermore, the authors determine in [4] the structure of such groups.

*Mathematics Subject Classification: 20E45, 20D10. Finite groups. Conjugacy class sizes. 
An evident question about solvability arises when we eliminate the coprime hypothesis and we are interested in studying which arithmetical conditions on groups with four class sizes yield their solvability. On the other hand, there seems to exist certain parallelism between the results obtained on the group structure from the set of its conjugacy class sizes and the results obtained from the set of its character degrees. In [10], groups whose character degrees are $\{1, m, n, m n\}$ are proved to be solvable. Our main result in this paper shows the same conclusion when the set of class sizes of a group $G$ is $\{1, m, n, m n\}$ where $m$ and $n$ are integers such that they do not divide mutually. In fact, we prove something else

Theorem A. Let $G$ be a finite group whose class sizes are $\{1, m, n, m n\}$, where $m, n$ are positive integers which do not divide one to another, then $G$ is up to central factors a $\{p, q\}$-group. In particular, $G$ is solvable.

We believe that the result is true for arbitrary integers $m$ and $n$, but we have not been able to prove it with the techniques we employ here.

Throughout this paper all groups are finite. If $x$ is any element of a group $G$, we denote by $x^{G}$ the conjugacy class of $x$ in $G$ and $\left|x^{G}\right|$ will be indistinctly called the conjugacy class size of $x$ or the index of $x$ in $G$. If $\pi$ is a set of primes and $G$ is a group, then $G_{\pi}$ will denote a Hall $\pi$-subgroup of $G$. All further unexplained notation is standard.

\section{Preliminary results}

We state the necessary results for the proof of our main theorem. The first result is elementary.

Lemma 1. Let $G$ be a finite group. A prime $p$ does not divide any conjugacy class size of $G$ if and only if $G$ has a central Sylow p-subgroup.

Proof. See for instance Theorem 33.4 of [8].

We will make use of the following interesting result due to Camina in the particular case in which $A_{0}=A$ is exactly the centralizer of an element of $G$ which moreover is both abelian and a maximal subgroup in the set of all proper centralizers in $G$.

Lemma 2. Let $G$ be a finite group with a subgroup $A_{0}$ such that $A_{0}$ is a characteristic subgroup of $A$, a subgroup of $G$, such that every element of $A_{0}$ has centralizer $A$ or $G$. Let $\pi$ be the set of primes dividing $\left|A_{0} / A_{0} \cap \mathbf{Z}(G)\right|$ and assume $|\pi|>1$. Then either

(i) $\mathbf{N}_{G}(A) / A$ is a $\pi^{\prime}$-group or

(ii) $\left|\mathbf{N}_{G}(A) / A\right|=p$ for some $p \in \pi$. 
Proof. This is Proposition 1 of [6].

The following result determines some properties of the structure of groups having exactly two conjugacy class sizes of $p$-regular elements for some prime $p$, that is, elements whose order is a $p^{\prime}$-number.

Theorem 3. Suppose that $G$ is a finite group and that $\{1, m\}$ are the $p$ regular conjugacy class sizes of $G$. Then $m=p^{a} q^{b}$, with $q$ a prime distinct from $p$ and $a, b \geq 0$. If $b=0$ then $G$ has abelian $p$-complement. If $b \neq 0$, then $G=P Q \times A$, with $P \in \operatorname{Syl}_{p}(G), Q \in \operatorname{Syl}_{q}(G)$ and $A \subseteq \mathbf{Z}(G)$. Furthermore, if $a=0$ then $G=P \times Q \times A$.

Proof. This result can be obtained as a consequence of two papers by A. Camina (see [7] and [?]). An easier proof can be found in Theorem A of [1].

Lemma 4. Let $K \unlhd G$, where $G$ is an arbitrary finite group and $K$ is abelian. Let $x$ be a noncentral element of $G$, and let $y=[t, x]$ for some element $t \in K$. Then $\left|\mathbf{C}_{G}(y)\right|>\left|\mathbf{C}_{G}(x)\right|$, and so the $G$-class of $y$ is smaller than that of $x$.

Proof. This is exacty Lemma 1 of [9].

Lemma 5. Let $x$ and $y$ be a q-element and a $q^{\prime}$-element respectively of a group $G$, such that $\mathbf{C}_{G}(x) \subseteq \mathbf{C}_{G}(y)$. Then $\mathbf{O}_{q}(G) \subseteq \mathbf{C}_{G}(y)$.

Proof. It is enough to apply Thompson's Lemma to the action of $\langle x\rangle \times\langle y\rangle$ on $\mathbf{O}_{q}(G)$.

\section{Proof of the main result}

In order to prove Theorem A, we will first prove the following particular case. In the following we will write $Z$ to denote the center of a group $G$.

Theorem 6. Let $G$ be a finite group whose class sizes are $\left\{1, p^{a}, n, p^{a} n\right\}$, where $p^{a}$ does not divide the positive integer $n$, then $G$ is up to central factors $a\{p, q\}$-group with $p$ and $q$ two distinct primes.

Proof. Notice that $p$ can be assumed to divide $n$, otherwise, the result follows by the main theorems of [2] and [3].

We assume first that there are no $p$-elements of index $p^{a}$. Notice that the centralizer of any element of index $p^{a}$ is a maximal subgroup among all centralizers, so by considering the primary decomposition we can assume that there exists some $q$-element $x$ of index $p^{a}$ for some prime $q \neq p$. If $y$ is a $q^{\prime}$-element of $\mathbf{C}_{G}(x)$, then $\mathbf{C}_{G}(x y)=\mathbf{C}_{G}(x) \cap \mathbf{C}_{G}(y) \subseteq \mathbf{C}_{G}(x)$, and since $p^{a}$ does not divide $n$, then $y$ has index 1 or $n$ in $\mathbf{C}_{G}(x)$. If there exists any $q^{\prime}$-element of $\mathbf{C}_{G}(x)$ of index $n$, then $n$ is the product of at most two prime powers by Theorem 3 , say 
$n=q^{b} r^{c}$. In this case, necessarily $r=p$, and then $G$ is a $\{p, q\}$-group (up to central factors by Lemma 1) and the proof is finished. If there is no $q^{\prime}$-element in $\mathbf{C}_{G}(x)$ of index $n$, then we can write $\mathbf{C}_{G}(x)=Q_{x} \times H_{x}$, with $H_{x}$ an abelian $q^{\prime}-$ complement of $\mathbf{C}_{G}(x)$. As $p$ divides $n$, it follows that $p^{a}<p^{a} n_{p} \leq|G: Z|_{p}$ and this yields the fact that $p$ divides $\left|\mathbf{C}_{G}(x) / Z\right|$. Hence, there exists a $p$-element in $\mathbf{C}_{G}(x)$, say $t$, such that $\mathbf{C}_{G}(x)=\mathbf{C}_{G}(t)$, so $t$ is a $p$-element of index $p^{a}$ and this is not possible.

Suppose now that there is a $p$-element $z$ of index $p^{a}$. Then, if $y$ is a $p^{\prime}$-element of $\mathbf{C}_{G}(z)$, we have $\mathbf{C}_{G}(z y)=\mathbf{C}_{G}(z) \cap \mathbf{C}_{G}(y)$. As in the above paragraph $y$ has index 1 or $n$ in $\mathbf{C}_{G}(z)$, and if there are $p^{\prime}$-elements of both indexes 1 and $n$ in $\mathbf{C}_{G}(z)$, it follows that $n=p^{a} q^{b}$ by Theorem 3. By Lemma 1 , we conclude that $G$ is a $\{p, q\}$-group (or a $p$-group if $b=0$ ) up to central factors. Suppose now that every $p^{\prime}$-element of $\mathbf{C}_{G}(z)$ has index 1 . Accordingly, we can write $\mathbf{C}_{G}(z)=P_{z} \times H_{z}$, where $H_{z}$ is an abelian $p$-complement. As $\mathbf{C}_{G}(z)$ has index $p^{a}$ in $G$, we deduce that $G$ has abelian $p$-complements, so in particular $G$ is solvable as it is the product of two nilpotent subgroups. Now, let us consider $\mathbf{O}_{p^{\prime}}(G)$. Since $\mathbf{O}_{p^{\prime}}(G)$ is an abelian normal subgroup of $G$, we can apply Lemma 4 and get $\left|\mathbf{C}_{G}([w, t])\right|>\left|\mathbf{C}_{G}(w)\right|$ for every $t \in G$ and for every $w \in \mathbf{O}_{p^{\prime}}(G)$ which is noncentral in $G$. Then, the hypotheses of the theorem imply that $\left[\mathbf{O}_{p^{\prime}}(G), G\right] \subseteq Z$. Moreover, by coprime action, we have $\mathbf{O}_{p^{\prime}}(G)=\mathbf{C}_{\mathbf{O}_{p^{\prime}}(G)}(P) \times$ $\left[\mathbf{O}_{p^{\prime}}(G), P\right]$, for any Sylow $p$-subgroup $P$ of $G$. Thus, $\left[\mathbf{O}_{p^{\prime}}(G), P\right]=1$ and, as the $p$-complements of $G$ are abelian, we deduce that necessarily $\mathbf{O}_{p^{\prime}}(G) \subseteq Z$, so $\mathbf{F}(G)=\mathbf{O}_{p}(G) \times Z_{p^{\prime}}$. Now, if $y$ is a $p^{\prime}$-element of $\mathbf{C}_{G}(z)$ which is non central in $G$, then $\mathbf{C}_{G}(z)=\mathbf{C}_{G}(y)$. By applying Lemma 5 , we obtain $y \in \mathbf{C}_{G}\left(\mathbf{O}_{p}(G)\right)=$ $\mathbf{C}_{G}(\mathbf{F}(G)) \subseteq \mathbf{F}(G)$, so $y \in Z$. Consequently, the $p$-complements of $G$ lie in $Z$, which is a contradiction.

Now, we are ready to prove our main result.

Proof of Theorem A. By Lemma 1, we can certainly assume that $\pi(G)=$ $\pi(m) \cup \pi(n)$. The proof is divided into 4 steps.

Step 1. If $x$ has index $m$, then $\mathbf{C}_{G}(x)$ is maximal among all centralizers. Also, either $\mathbf{C}_{G}(x)$ is abelian or $n=p^{a} q^{b}$ for some primes $p$ and $q$, and $\mathbf{C}_{G}(x)=$ $P_{x} Q_{x} \times T_{x}$, where $P_{x}$ and $Q_{x}$ are $p$ and $q$ subgroups respectively, and $T_{x}$ is an abelian $\{p, q\}^{\prime}$-group. The same properties can be assumed for all elements of index $n$.

Suppose that $x$ has index $m$, and hence it can be assumed to be a $p$-element for some prime $p$. Notice that $\mathbf{C}_{G}(x)$ is a maximal subgroup among all centralizers. Since every $p^{\prime}$-element of $\mathbf{C}_{G}(x)$ has index 1 or $n$ in $\mathbf{C}_{G}(x)$ we have two possibilities as we have already explained in the proof of Theorem 6: either $\mathbf{C}_{G}(x)=P_{x} \times H_{x}$ with $H_{x}$ an abelian $p^{\prime}$-subgroup or $n=p^{a} q^{b}$ for some prime $q$ and $\mathbf{C}_{G}(x)=P_{x} Q_{x} \times T_{x}$ as described in the statement. We will show that the first possibility also yields to the statement of this step. If $H_{x} \subseteq Z$, then $|G: Z|=m p^{d}$ and since any class size divides this index, we deduce that $n$ is a 
power of $p$, so our claim is proved. Therefore, for some prime $q$ we can take some noncentral $q$-element $t \in H_{x}$ and certainly $\mathbf{C}_{G}(x)=\mathbf{C}_{G}(t)$. But this implies again that any $q^{\prime}$-element of $\mathbf{C}_{G}(t)$ has index 1 or $n$. Hence, we have either $\mathbf{C}_{G}(t)=Q_{t} \times K_{t}$ with $K_{t}$ an abelian $q$-complement, and accordingly $\mathbf{C}_{G}(x)$ is abelian, or $n$ is again a product of two prime powers, as wanted.

Now if $y$ has index $n$ then $\mathbf{C}_{G}(y)$ is maximal among all centralizers as well and the assertion in the statement of the other property for $\mathbf{C}_{G}(y)$ follows exactly as for $\mathbf{C}_{G}(x)$.

Step 2. We can assume that there exists some element of index $m$ or $n$ such that its centralizer is not abelian. We can fix a $q$-element $x$ of index $m$ such that $\mathbf{C}_{G}(x)$ is not abelian and $\mathbf{C}_{G}(x)=P_{x} Q_{x} \times Z_{\{p, q\}^{\prime}}$, where $P_{x}$ and $Q_{x}$ are $p$ and $q$ subgroups and such that $x$ is a $q$-element. Moreover, $n=p^{a} q^{b}$.

Suppose that all elements of index $m$ and $n$ have abelian centralizer and put $A=\mathbf{C}_{G}(x)$ and $B=\mathbf{C}_{G}(y)$ for some elements $x$ and $y$ of index $m$ and $n$ respectively. If $u \in A \cap B$, then $A, B \leq \mathbf{C}_{G}(u)$, whence $\left|G: \mathbf{C}_{G}(u)\right|<\min \{m, n\}$ and so $u \in Z$. Therefore, $A \cap B=Z$. Then

$$
|A / Z|=|A / A \cap B|=|A B| /|B| \leq|G: B|=n
$$

and so

$$
|G / Z|=|G: A||A / Z| \leq m n .
$$

But this certainly contradicts the fact that $G$ has elements of index $m n$

Thus, we will assume that there exists some element in $G$ of index $m$ or $n$ whose centralizer is not abelian. By the symmetry of the hypothesis, we can fix some $x$ of index $m$. By Step 1, we have $\mathbf{C}_{G}(x)=P_{x} Q_{x} \times T_{x}$, where $P_{x}$ and $Q_{x}$ are $p$ and $q$ subgroups, and $T_{x}$ is an abelian $\{p, q\}^{\prime}$-group. Furthermore, $n=p^{a} q^{b}$. We prove that $T_{x}$ must be central in $G$. In fact, if there exists some noncentral $r$-element $t \in T_{x}$ for some prime $r \neq q, p$ then, since $\mathbf{C}_{G}(x) \subseteq \mathbf{C}_{G}(t)$, they are equal by the maximality. Also, every $r^{\prime}$-element of $\mathbf{C}_{G}(t)$ has index 1 or $n$. Since $r$ is not a divisor of $n$, by Theorem 3 we obtain that $n$ is either a power of $p$ or $q$, and then the proof of the theorem finishes by Theorem 6 . Furthermore, by using the maximality of $\mathbf{C}_{G}(x)$, we can also assume without loss that $x$ is has prime power order, and without loss, for instance, that it is a $q$-element.

Step 3. If $z$ is an element of index $m n$, then $\mathbf{C}_{G}(z)=P_{z} Q_{z} \times Z_{\{p, q\}^{\prime}}$, where $P_{z}$ is a $p$-subgroup and $Q_{z}$ is a $q$-subgroup of $\mathbf{C}_{G}(z)$ both noncentral in $G$, and $Z_{\{p, q\}^{\prime}}$ denotes the $\{p, q\}$-complement of $Z$. As a consequence, we have that both $p$ and $q$ divide $\left|\mathbf{C}_{G}(y) / Z\right|$ for every element $y$ of index $n$.

Let $z$ be an element of index $m n$. If $r$ divides $\left|\mathbf{C}_{G}(z) / Z\right|$ and $r \neq p, q$, then $|G / Z|_{r}>(m n)_{r} \geq m_{r}$, but $m_{r}=|G / Z|_{r}$ by Step 2. Thus we can write $\mathbf{C}_{G}(z)=P_{z} Q_{z} \times Z_{\{p, q\}^{\prime}}$, with $P_{z}$ a $p$-subgroup and $Q_{z}$ a $q$-subgroup of $\mathbf{C}_{G}(z)$. 
We will prove now that both $P_{z}$ and $Q_{z}$ cannot be central in $G$. Suppose that $Q_{z}$ is central in $G$. Since $p$ divides $\left|\mathbf{C}_{G}(x) / Z\right|$, where $x$ is the element fixed in Step 2, there exists some $p$-element in $\mathbf{C}_{G}(x) \backslash Z$. Let us take any noncentral $p$-element, say $w$, in $\mathbf{C}_{G}(x)$. Notice that $w x$ cannot have index $m n$, otherwise $\left|\mathbf{C}_{G}(w x) / Z\right|=\left|\mathbf{C}_{G}(z) / Z\right|$ is a $p$-power and this is not possible because $x \in \mathbf{C}_{G}(w x) \backslash Z$. Thus $\mathbf{C}_{G}(w x)=\mathbf{C}_{G}(x)=\mathbf{C}_{G}(w)$, and every $q^{\prime}-$ element, so in particular every $p$-element, of $\mathbf{C}_{G}(x)$ is central in $\mathbf{C}_{G}(x)$. Hence $\mathbf{C}_{G}(x)=P_{x} \times Q_{x} \times Z_{\{p, q\}^{\prime}}$ with $P_{x}$ abelian. Likewise, if $t$ is a $q$-element of $\mathbf{C}_{G}(w)$, similarly we get $\mathbf{C}_{G}(t w)=\mathbf{C}_{G}(w)=\mathbf{C}_{G}(t)$ and $\mathbf{C}_{G}(w)=\mathbf{C}_{G}(x)$ has an abelian Sylow $q$-subgroup too. This shows that $\mathbf{C}_{G}(x)$ is abelian, which is a contradiction.

Observe that $P_{x}$ is not central, otherwise Step 2 would imply that $n$ is a prime power and the theorem is true by Theorem 1 . Now let $t$ be a $p$-element in $\mathbf{C}_{G}(x) \backslash Z$. If $P_{z}$ is central in $G$ then, arguing as in the above paragraph, we have that $x t$ cannot have index $m n$ and so $\mathbf{C}_{G}(x t)=\mathbf{C}_{G}(t)=\mathbf{C}_{G}(x)$. We deduce again that $\mathbf{C}_{G}(t)=\mathbf{C}_{G}(x)$ is abelian, a contradiction, so the step is proved.

As we know that $n$ and $m$ are not coprime numbers, from now on we will assume that $p$ is a common prime of $m$ and $n$ (we argue similarly if the common prime is $q$ ).

Step $4 . G$ is a $\{p, q\}$-group.

Let $y$ be an element of index $n$. We are going to show that we can assume that $\mathbf{C}_{G}(y)$ is not abelian. Suppose that $\mathbf{C}_{G}(y)$ is abelian. First, we will assume that $\mathbf{C}_{G}(y)=\mathbf{N}_{G}\left(\mathbf{C}_{G}(y)\right)$ and we will get a contradiction. We know that $p$ divides $\left|\mathbf{C}_{G}(y) / Z\right|$ by Step 3. Moreover, as $\mathbf{C}_{G}(y)$ is abelian and by the maximality of this centralizer, there exists some $p$-element $t \in \mathbf{C}_{G}(y)$ such that $\mathbf{C}_{G}(t)=\mathbf{C}_{G}(y)$. Since $p$ divides $m$ and $n$, we have that the Sylow $p$-subgroups of $G$ are not abelian. Let $P$ be a Sylow $p$-subgroup of $G$ such that $t \in P$ and notice that $\mathbf{Z}(P)=Z_{p}$. We can take $\bar{z} \in \mathbf{Z}\left(P / Z_{p}\right)$ and then $\bar{z} \bar{t}=\bar{t} \bar{z}$. Whence $z \in \mathbf{N}_{G}\left(\mathbf{C}_{G}(t)\right)=\mathbf{C}_{G}(t)$. Thus, $z \in \mathbf{C}_{G}(y)$. By the maximality of $\mathbf{C}_{G}(y)$ and using the fact that $\mathbf{C}_{G}(y)$ is abelian again, we conclude that $\mathbf{C}_{G}(y)=\mathbf{C}_{G}(z)$. However, $P \subseteq \mathbf{N}_{G}\left(\mathbf{C}_{G}(z)\right)=\mathbf{C}_{G}(z)$, but this is not possible, as wanted.

Therefore, by arguing as in the above paragraph, we can take a $p$-element $z \in$ $\mathbf{N}_{G}\left(\mathbf{C}_{G}(y)\right) \backslash \mathbf{C}_{G}(y)$, whence $p$ divides $\left|\mathbf{N}_{G}\left(\mathbf{C}_{G}(y)\right) / \mathbf{C}_{G}(y)\right|$. However, by using Lemma 2 and taking $A=A_{0}=\mathbf{C}_{G}(y)$ we obtain exactly $\left|\mathbf{N}_{G}\left(\mathbf{C}_{G}(y)\right) / \mathbf{C}_{G}(y)\right|=$ $p$.

On the other hand, we know that $p$ and $q$ both divide $\left|\mathbf{C}_{G}(y) / Z\right|$ by Step 3 . Since $q$ divides $n$, if $Q_{y}$ is the Sylow $q$-subgroup of $\mathbf{C}_{G}(y)$, then $Q_{y}<Q$ for some Sylow $q$-subgroup $Q$ of $G$. If $v \in \mathbf{N}_{Q}\left(Q_{y}\right) \backslash Q_{y}$, then $Q_{y} \subseteq \mathbf{C}_{G}(y) \cap \mathbf{C}_{G}\left(y^{v}\right)$. As $\mathbf{C}_{G}(y)$ is abelian, we deduce that $\mathbf{C}_{G}(y)=\mathbf{C}_{G}\left(y^{v}\right)=\mathbf{C}_{G}(y)^{v}$. This means that $v$ is a $q$-element lying in $\mathbf{N}_{G}\left(\mathbf{C}_{G}(y)\right) \backslash \mathbf{C}_{G}(y)$, but this cannot occur.

Thus, $\mathbf{C}_{G}(y)$ is not abelian, as wanted. By using Steps 1 and 3, we conclude that $m=p^{c} q^{d}$. Consequently, $m$ and $n$ are $\{p, q\}$-numbers and $G$ is a $\{p, q\}$ group. 
This research is supported by the Spanish Government, Proyecto MTM201019938-C03-02 and by the Valencian Government, Proyecto PROMETEO/2011/30, and the first author is also supported by grant Fundació Caixa-Castelló P11B201047.

\section{References}

[1] E. Alemany, A. Beltrán, M.J. Felipe, Finite groups with two p-regular conjugacy class lengths II, Bull. Aust. Math. Soc., 79 (2009) 419-425.

[2] A. Beltrán, M.J. Felipe, Variations on a Theorem by Alan Camina on conjugacy class sizes. J. Algebra 296 (2006) 253-266.

[3] A. Beltrán, M.J. Felipe, Some class size conditions implying solvability of finite groups. J. Group Theory 9 (2006) 787-797.

[4] A. Beltrán, M.J. Felipe, Finite groups with four conjugacy class sizes. Comm. in Algebra 39 (4) (2011) 1260-1272.

[5] A.R. Camina, R.D. Camina, Coprime Conjugacy Class Sizes, Asian-Eur. J. Math. 2 (2), (2009) 183-190.

[6] A.R. Camina, Finite groups of conjugate rank 2, Nagoya Math. J. 53 (1974), 47-57.

[7] A.R. Camina, Conjugacy classes of finite groups and some theorems of N.Itô. J. London Math. Soc. 6 (2) (1973) 421-426.

[8] B. Huppert, Character Theory of Finite Groups, De Gruyter Expositions in Mathematics 25, Walter de Gruyter \& Co, Berlin, 1998.

[9] I.M. Isaacs, Subgroups generated by small classes in finite groups. Proc. Amer. Math. Soc. 136, 2299-2301 (2008).

[10] G. Qian, W. Shi, A note on character degrees of finite groups. J. Group Theory 7 (2004), 187-196. 